This item was submitted to Loughborough's Research Repository by the author.

Items in Figshare are protected by copyright, with all rights reserved, unless otherwise indicated.

\title{
Tilt control design for high-speed trains: a study on multi-objective tuning
} approaches

PLEASE CITE THE PUBLISHED VERSION

PUBLISHER

(c) Taylor \& Francis

VERSION

AM (Accepted Manuscript)

LICENCE

CC BY-NC-ND 4.0

\section{REPOSITORY RECORD}

Zamzuri, Hairi, Argyrios C. Zolotas, and Roger M. Goodall. 2019. "Tilt Control Design for High-speed Trains: A Study on Multi-objective Tuning Approaches". figshare. https://hdl.handle.net/2134/5506. 
This item was submitted to Loughborough's Institutional Repository (https://dspace.lboro.ac.uk/) by the author and is made available under the following Creative Commons Licence conditions.

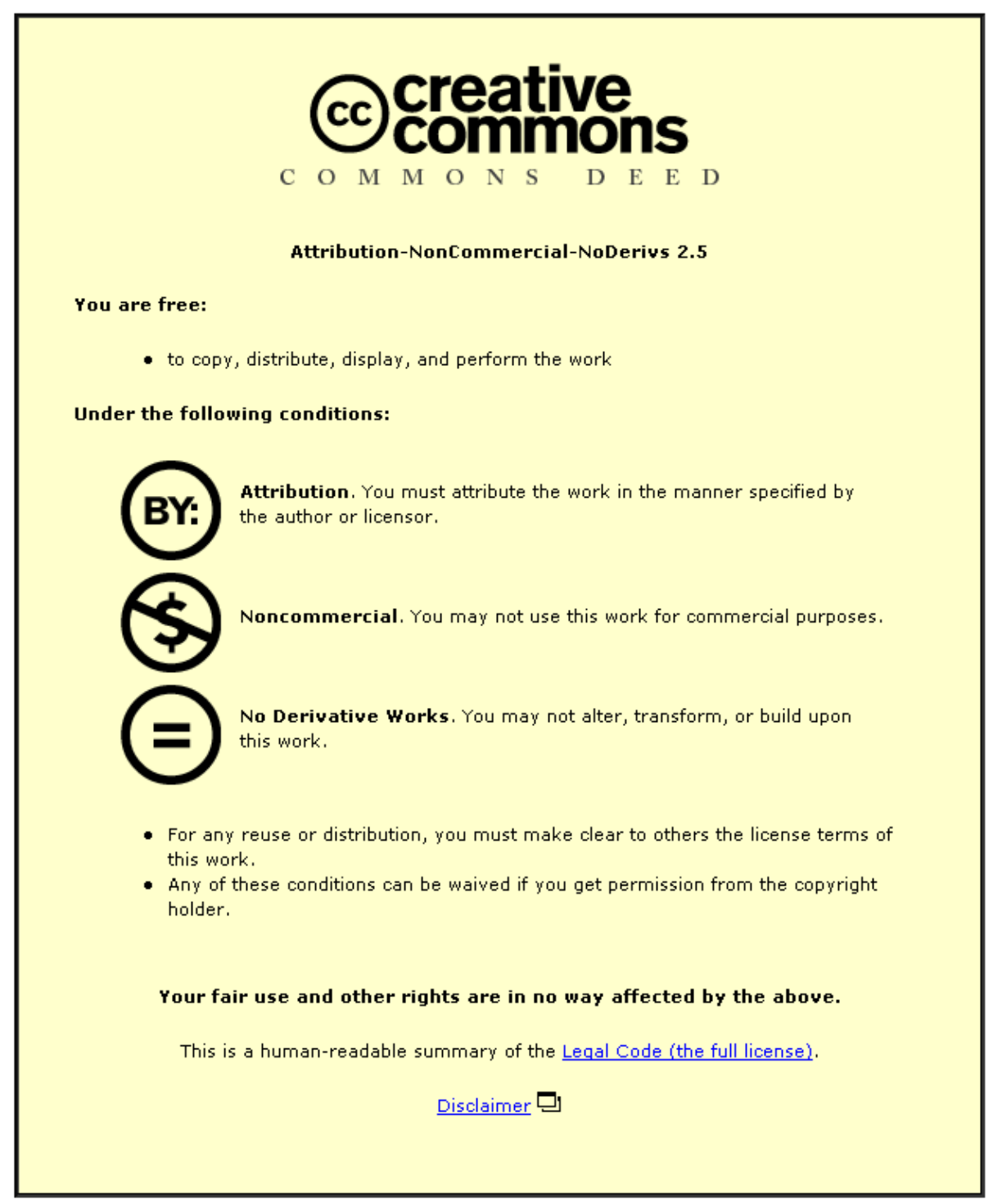

For the full text of this licence, please go to: http://creativecommons.org/licenses/by-nc-nd/2.5/ 


\title{
TILT CONTROL DESIGN FOR HIGH SPEED TRAINS: A STUDY ON MULTI OBJECTIVE TUNING APPROACHES.
}

\author{
Hairi Zamzuri* $\dagger$, Argyrios C. Zolotas $\dagger$, Roger M. Goodall $\dagger$ \\ $\dagger$ Department of Electronic and Electrical Engineering, Loughborough University, UK
}

\begin{abstract}
This paper presents work on a hybrid fuzzy control scheme for improving the performance of tilting trains using a nulling-based tilt strategy. Two multi-objective Genetic Algorithm tuning methods (MOGA and NSGAII) were employed to optimise both the fuzzy output membership functions and the controller parameters. The objective functions incorporated the tilt response and roll gyroscope signals for the deterministic (curved track) profile, and lateral acceleration for the stochastic (straight track) profile. Simulation results discuss the effectiveness of using the presented techniques for tuning the fuzzy control scheme via multiple objectives. The proposed scheme is compared with the conventional nulling-tilt approach and a manually-tuned fuzzy controller.
\end{abstract}

Keywords: railway dynamics; tilt; intelligent control; tilting trains; fuzzy control; multi objective genetic algorithm

\section{Introduction}

High-speed trains can substantially reduce journey times and the way to achieve this is either to develop new infrastructure that maximises trains speeds or to use existing infrastructure with the introduction of tilting trains. Building new infrastructure is usually a very expensive option and issues related to the surrounding environment arise. Tilting train technology follows a rather straightforward concept, i.e by leaning the vehicle body inwards on curved section and thus reduce the lateral acceleration pressure on passengers, thereby enabling higher vehicle speeds.

Early tilting train applications used a classical nulling-tilt approach based on local vehicle measurements; however it was found impossible at the time to get an appropriate solution (including both performance and stability problems) [7]. Current tilting trains use the so-called 'precedence' tilt control strategy, whereby a bogie-mounted accelerometer from the vehicle in front is used to provide "precedence" or preview track information (via appropriate inter-vehicle cable/signalling connections), carefully designed so that the delay introduced by the filter compensates for the preview time corresponding to approximately a vehicle length. However, this approach is not strictly optimized from a system point of view, while achieving a satisfactory local tilt control strategy (i.e based only upon measurements from the vehicle itself) remains an important research topic due to system simplifications and more straightforward failure detection. For both tilt schemes see Fig. 1.

Work on modern advanced control schemes for improving the local tilt vehicle performance is presented by [16]. Results on the feasibility of using fuzzy logic controllers for tilt performance improvement have been presented in Zamzuri et al [14], which also introduced a fuzzy-correction tilt control scheme. In this scheme, the tuning process of the overall controller structure is often achieved manually through trial and error procedure which can be time-consuming, with designer experience being a key issue. An improved tuning method described in Zamzuri et al [15] optimized the parameters by using a simple genetic algorithm with weighting factors for combining the different objective, but it proved difficult to assign values of each weight. This paper presents work on an extension of the fuzzy-correction control scheme by introducing two multi-objective tuning approaches known as Non-Domination Sorting Genetic Algorithm (NSGA-II) and Multi-Objective Genetic Algorithm (MOGA).

*The corresponding author is also attached to College of Sciences and Technologies, Universiti Teknologi Malaysia, Kuala Lumpur, Malaysia. Email : h.zamzuri@lboro.ac.uk, hairi@citycampus.utm.my

\section{Vehicle System Dynamics}

Author final version (Vehicle System Dynamics, 2008, 46:1, pp. 535-547) 
The paper is organized as follows: Section 2 briefly describes the vehicle model characteristics used in this work. The performance assessment of the controller schemes is presented in Section 3. Section 4 discusses the Multi-Objective Genetic algorithm concepts, while the fuzzy control design is presented in Section 5 . More details on the multi-objective tuning approach for the purpose of the tilting train study are discussed in Section 6. Simulation results and related analysis are presented in Section 7. Finally, conclusions are drawn in Section 8.

\section{Vehicle Model}

The vehicle model is based upon a linearised end-view diagram shown in Fig.2, which includes the lateral and roll dynamics of both the body and the bogie, plus the dynamic contribution of the air spring.

A pair of linear air springs represents the secondary suspensions, which only contribute to the roll motion of the vehicle. The model also includes the stiffness of an anti-roll bar connected between the body and the bogie frames. Active tilt is provided via a rotational displacement actuator, included in series with the roll stiffness (the concept of an 'active anti-roll bar (ARB)' [10]). The active anti roll bar is assumed to cater for up to a maximum tilt angle of 10 degrees. The advantages of active ARBs results from their relative simplicity, i.e. small weight increase, low cost, easily fitted as an optional extra to build or as a retro-fit. It is worth noting that the system is rather complex and characterised by significant coupling between the lateral and roll motion; the dynamic modes which result are often referred to as "sway modes". More details regarding the mathematical description can be found in Zolotas et al [17].

\section{Performance Assessment}

Two main design criteria are concerned with tilting trains: (i) deterministic criterion: provide a fast response on curved track, (ii) stochastic criterion: maintain an acceptable ride quality in response to track irregularities on straight track segments. The performance assessment approach proposed in Goodall et al [8] was utilised for the purposes of this work.

The assessment on curve transitions relates to the idea of "ideal tilting", i.e. whereby the tilt action follows the specified tilt compensation in an ideal manner according to the maximum tilt angle and cant deficiency compensation factor. Deviation from the "ideal tilting" response quantifies the additional dynamic effects caused by the suspension/controller dynamics during the transitions to and from the curves, and provides an objective measure which can be used to compare different strategies.

The straight track performance criterion is based on the RMS lateral ride quality in response to the effect of track irregularities, typically no more than $7.5 \%$ degradation. For proper comparison this is made at high speed for both the tilting and non-tilting cases.

However most tilt schemes act through or across the secondary suspension and only indirectly effect the vehicle lateral acceleration. Thus, a fundamental trade-off exists between the vehicle curve transition response and straight track performance. In addition, for reasons of human perception, designers employ partial tilt compensation, whereby the passenger still experiences a small amount of acceleration on steady curves in order to minimize motion sickness phenomena.

\section{Multi-Objective GA Optimization}

Genetic Algorithms (GAs) have been successfully applied to automatic control design in various areas of control engineering [2], [11]. Initially they were applied almost exclusively to a single objective problem but most of the real-life engineering applications require several objectives to be optimized.

A single objective optimization problem seeks to obtain the best solution. However, in multi-objective problems more than one (best) solution may exist with respect to all objectives. For a typical multiobjective problem, there exists a set of solutions which may be superior to others solutions in the search space considering all objectives, but inferior to other solutions in the space where only one or few objectives 
are considered. These are known as Pareto-optimal or non-dominated solutions. Early multi-objective solutions scalarized the vector of objectives into one objective by using averaging methods with a weight vector. This solution might be simple and easy to use but obtaining an appropriate result largely depends on the selection of the weights. Schaffer [12] proposed the Vector Evaluated Genetic Algorithm (VEGA) which differs from the Simple Genetic Algorithm in the selection process. This operation was modified so that at each, generation, a number of sub-populations was generated by performing proportional selection according to each of the objective functions.

Pareto-based fitness assignment was first proposed by Goldberg [6], searching a set of chromosomes in the population that are pareto non-dominated by the rest of the populations. Horn et al [9] extended the tournament method to a multiple optimization genetic algorithm problem known as Niched Pareto Genetic Algorithm (NPGA). Another method called Nondominated Shorting Genetic Algorithm (NSGA) is based on several layers of classification of individuals, where the population is ranked on the basis of non-dominated solutions before selection is performed [13].

Fonseca and Fleming [4,5], proposed the Multiple Objective Genetic Algorithm (MOGA) in which the ranking of the individuals corresponds to the number of populations by which it is dominated. This method employs the principle of pareto-dominance as the basic of assigning a scalar fitness value to a vector objective function. Rank-based selection and niched methods are employed to generate populations of a non-dominated solution estimated without having to combine the objectives.

In fact, the rank-based scheme proposed by Fonseca and Fleming assigns all the non-dominated individuals to rank ' 1 ', while dominated individuals are penalized according to the population density of the corresponding region of trade-off. The improvement of the non-dominated sorting genetic algorithm (NSGAII) by Deb et al [3] is based on the principles of the previous method. Moreover, the crowding distance is used to estimate the density of the solutions surrounding each individual on a given front. In the tournament selection method, if the individuals belong to the same front, the selection is based on the front with a greater crowding distance. All details of the algorithm can be found in the paper [3].

\section{$5 \quad$ Fuzzy Control design}

The control design objective is to provide fast responses on curved track while minimizing the tilt system's adverse response to track irregularities on straight segments of the track. The controller approach followed in this paper involves the design of a conventional PID controller to give a fast curve transition performance and a fuzzy-correction mechanism which improves stability (minimize overshoots and prevent critical oscillations) in the overall system. The control scheme can be seen in Fig. 3. The PID controller is driven by the effective cant deficiency signal guaranteeing the appropriate tilt compensation on a steady curve (i.e. $60 \%$ in this work). Moreover, the output signal from the PID ( $\left.u^{\prime}\right)$ is the input to the fuzzy correction block to further accommodate for curve transition and straight track performance. The body roll rate is fed back to the fuzzy correction block and acts as an additional decision-making variable. Thus, the design of the controller can be divided into three stages:

(i) conventional PID to give fast, accurate response on curved track

(ii) fuzzy correction aimed at optimising the responses to straight track irregularities and preventing large overshoot and oscillations on curve transitions.

(iii) further tuning using multi-objective GA (MOGA and NSGA II in this case)

In the first instance the PID can be tuned based on the well-known Ziegler-Nichols method [1]. The parameters are chosen to give the fastest possible response subject to guaranteeing stability.

Both inputs to the fuzzy correction mechanism shown in Fig. 4(a), consist of three equally distributed Gaussian Membership Functions with 50\% overlap for each signal. In addition, Fig. 4(b) shows the fuzzy correction output $u$ " consisting of trapezoidal and triangle membership functions. The Center of Area (COA) defuzzification procedure with the well known max-min inference method used to formulate the fuzzy algorithm. 
The linguistic variables for each membership functions represent the condition for each value. The input membership functions from the PID output $u$ ' and the body roll gyro input $\dot{\theta}_{v}^{\text {gyro }}$ are represented by the linguistic variables $N e g$, Zero and Pos. For the fuzzy correction output $u$ " the linguistic variables represent the tilting direction of the car body, for example tilt clockwise maximum is donated by TiltClkwM, tilt medium anticlockwise by TiltAclkwm etc. Clockwise and Anticlockwise denote the direction of tilt based on the curve direction (i.e inwards and outwards is relative to the direction of the curve respectively). Note that the membership function ranges represent the required operating range of the variables. The development of fuzzy rules was based on the following heuristic statements:

- stabilizing the system:

if $u^{\prime}$ is changing fast and the $\dot{\theta}_{v}^{\text {gyro }}$ is zero then apply maximum tilt effort u"

- preventing overshoot and oscillation.

\section{if u' changes and $\dot{\theta}_{v}^{g y r o}$ changes then maintain medium tilt effort u"}

with more details on the rules shown in Table 1.

\section{Multi-Objective GA Tuning design}

There is a strict trade-off in designing local tilt controllers for high speed trains, in terms of providing fast curved transition response and maintaining straight track ride quality. In this paper, eight real-coded ${ }^{1}$ GA variables were developed to optimize the PID parameters $\left(K_{P}, K_{D}\right.$ and $\left.K_{I}\right)$, the position and width of output fuzzy membership functions (see Fig 3). The upper and lower limits on the parameters are set based on experience of manually tuning the PID Fuzzy controller, see [14]. Appropriate initial choice of the parameters and the limits undoubtedly reduces the computational time and complexity.

In this study only the output membership function has been used for the optimization process to avoid additional tuning parameters, and thus increased complexity. Fig. 4(b) presents the output membership function which consists of three triangular membership functions and two trapezoidal membership functions located at each end of the fuzzy set. Fig. 5 illustrates the concept of coding the membership functions. The GA seeks the optimal profile (based on position and width of the membership functions), except for the center of the membership function NoChange and those at the limit of the range (TiltClkwM , TiltAclkwm). Note, the membership functions are symmetrical for both side except for the NoChange membership function.

Six objectives, $f_{x}$ with constraints,$\omega_{x}$ were used:

(i) deterministic: settling time at steady state curve, $t_{s}$ not more than $5 \mathrm{sec}$

$$
f_{1}=t_{s}
$$

where the constraint function is given by

$$
\omega_{1}=\left\{\begin{array}{l}
f_{1} \text { if } f_{1}>5 \mathrm{sec} \\
0 \text { otherwise }
\end{array}\right.
$$

(ii) deterministic: optimize the curved track response based on maximum peak lateral deviation error between ideal $\ddot{y}_{m_{i}}$ and actual $\ddot{y}_{m}$ lateral acceleration taken between $1 \mathrm{sec}$ before and $3.6 \mathrm{sec}$ after the start and end of transition for both $\operatorname{in}\left(f_{2}\right)$ and out $\left(f_{3}\right)$ of the curved transition track, given by :

$$
f_{(2,3)}=\left|\ddot{y}_{m i(2,3)}-\ddot{y}_{m(2,3)}\right|_{\text {peak }}
$$

\footnotetext{
${ }^{1}$ real-coded GA is referred to the real numbers representing the actual parameter values in order to form the chromosome structure.
} 
and the constraint function is given by,

$$
\omega_{(2,3)}= \begin{cases}f_{(2,3)} & \text { if } f_{(2,3)}>5.5 \% \mathrm{~g} \\ 0 & \text { otherwise }\end{cases}
$$

(iii) deterministic: optimize the body roll rate angle response to system changes based on the RMS (root mean square) deviation error between the actual $\dot{\theta}_{m}$ and ideal roll rate $\dot{\theta}_{m_{i}}$ profile. The calculation is taken from $1 \mathrm{sec}$ before until $3.6 \mathrm{sec}$ after the start and at the end of curve transition for both in $\left(f_{4}\right)$ and $\operatorname{out}\left(f_{5}\right)$ of the curved transition track.

$$
f_{r m s(4,5)}=\sqrt{\frac{1}{T_{2(4,5)}-T_{1(4,5)}} \int_{T_{1(4,5)}}^{T_{2(4,5)}}\left|\dot{\theta}_{m i_{(4,5)}}-\dot{\theta}_{m_{(4,5)}}\right|^{2}} d t
$$

where $T_{1}$ and $T_{2}$ is the respective curve transition time, and the constraint function,

$$
\omega_{x}= \begin{cases}f_{(4,5)} & \text { if } f_{(4,5)}>0.03 \mathrm{rad} / \mathrm{s} \\ 0 & \text { otherwise }\end{cases}
$$

(iv) stochastic $f_{6}$ : constrain the degradation of the straight track ride quality within the allowed degradation of $7.5 \%$ taken between the active (with controller) and passive (without controller) system at high speed.

$$
\omega_{6}=\left\{\begin{array}{l}
f_{6} \text { if } f_{6}>7.5 \% \\
0 \text { otherwise }
\end{array}\right.
$$

The constraint violation functions are added together to give the overall constraint, given by

$$
\Omega_{x}=\sum_{x=1}^{6} \omega_{x}
$$

Therefore the objective function with constraint is given by

$$
F_{m}(x)=f_{x}+R_{x} \Omega_{x}
$$

where $R_{x}$ is the penalty parameter and $x=1,2 . .6$.

Figure 6 shows the graphical responses of the overall calculations.

Multi-Objective Genetic algorithm (MOGA) [5] and Fast and Elitist Multi-Objective Genetic Algorithm (NSGA II) [3] were used to optimize the parameters of PID and Fuzzy mechanism. For comparison purposes, both methods used the same objective functions proposed in this section.

\section{$7 \quad$ Result and analysis}

SIMULINK was used to simulate the overall system, while the GA procedure was followed and implemented in MATLAB. A random initial population of size 30 and shuffle crossover with binary mutation were used with crossover and mutation probability of 0.9 and 0.01 respectively for both optimization searching methods. Due to the limited space, Fig. 8 shows only the comparison between ride degradation $f_{6}$, and in-curve peak lateral deviation, $f_{2}$. Overall, the figure shows the tuning process towards a better result. Fig. 7(d) reveals the use of NSGAII has a better population diversity compared to the MOGA method. 
Moreover, the system was simulated at a speed of $210 \mathrm{~km} / \mathrm{h}$ using a $1000 \mathrm{~m}$ curved radius and $6^{\circ}$ track cant angle (the track profile included $145 \mathrm{~m}$ transition length at each end of the curve). For comparison, four different controller structures and tuning were compared:

(i) PID with fuzzy correction mechanism (manually tuned [14])

(ii) and the same controller type as 1) tuned via MOGA

(iii) and NSGAII

(iv) a conventional nulling PI controller.

Fig. 8 illustrates the resultant output membership function for the manually tuned and the optimized using multi objective genetic algorithms. Table 2 compares the parameter values of the PID controller from the Ziegler-Nichols tuning method and the proposed genetic algorithms schemes.

The time domain results for lateral acceleration, body roll angle and body roll rate profiles can be seen in Fig. 9(a), Fig. 9(b) and Fig. 9(c) respectively. It is clearly seen that the fuzzy-based schemes improve the performance substantially (closer to the ideal case). The multiobjective GA solution offers a rather noticeable improvement compared to the manually-tuned scheme. From the application point of view, both tuning processes (MOGA and NSGAII) can be used; however, using NSGAII gives a multiple choice compared to MOGA method.

Table 3 presents the overall performance assessment (using the method discussed in section 3 ) of the system using four mentioned controllers. The performance improvement is clearly seen using the fuzzy schemes. The effectiveness of the multiobjective GA tuning schemes is also shown more clearly, providing further performance improvement in an otherwise nulling-based design.

\section{Conclusion}

The paper illustrates the potential of using a PID-Fuzzy with the assistance of multi objective tuning approaches. Two tuning approaches have been used and analyzed to investigate the capability of these approaches. A comparison between the GA tuned schemes, a manually tuned fuzzy equivalent and the conventional classical nulling tilt approach clearly shows the advantages of using the former solution. The multiobjective approaches offers a more straightforward tuning procedure via optimization, subject to appropriately formulating the problem, compared to a usually time-consuming manually-tuned design. In addition, a number of design objectives can be simultaneously tackled via this approach. The objective functions for the deterministic profile were based on the tilt response and body roll rate signals, while stochastic straight track ride quality was constrained by using the lateral acceleration signal.

Current work utilises fuzzy-correction combined with modern model-based controller.

\section{References}

[1] K. Astrom and T. Hugglund. PID controllers: Theory, Design, and Tuning $2^{\text {nd }}$ Ed. Inst. Society of America, 1995.

[2] A. Chipperfield and P. Fleming. Multiobjective gas turbine engine controller design using genetic algorithms. Industrial Electronics, IEEE Transactions on, 43(5):583-587, 1996.

[3] K Den, A Pratap, S Agarwal, and T Meyarivan. A fast and elitist multiobjective genetic algorithm: NSGA II. Evolutionary Computation,IEEE Transaction on, 6(5):183-197, 2002.

[4] C. M. Fonseca and P. J. Fleming. Multiobjective optimal controller design with genetic algorithms. In Control, 1994.Control '94.Volume 1., International Conference on, pages 745-749 vol.1, 1994.

[5] C.M Fonseca and P.J. Fleming. Genetic Algorithm for Multiobjective Optimization: Formulation, Discussion and Generalization. In Proceedings of the fifth International Conference on Genetic Algorithms, pages 416-423, San Mateo, CA, 1993. S. Forrest Ed.

[6] D.E. Golberg. Genetic algorithm in Search, Optimization and Machine Learning. Addison-Wesley, USA, 1989.

[7] R. M. Goodall. Tilting trains and beyond - the future for active railway suspensions: Part 1 Improving passenger comfort. Computing and Control Enginerring Journal, 10(4):153-160, 1999.

[8] R. M. Goodall, A. C. Zolotas, and J. Evans. Assesment of the performance of tilt system controllers. In The Railway Conference at Railtex 2000, pages 21-23, NEC Birmingham, UK, 2000.

[9] J. Horn, N. Nafpliotis, and D. E. Goldberg. A Niched Pareto Genetic Algorithm for multiobjective optimization. In Evolutionary Computation, 1994.IEEE World Congress on Computational Intelligence., Proceedings of the First IEEE Conference on, volume 1, pages $82-87,1994$.

[10] J. T. Pearson, R. M. Goodall, and I. Pratt. Control system studies of an active anti-roll bar tilt system for railway vehicles. Proceedings Inst of Mechanical Engineering, 212 Part F:43-60, 1998.

[11] A. Popov, A. Farag, and H. Werner. Tuning of a PID controller using a Multi-objective Optimization Technique Applied to a 
Neutralization Plant. In Decision and Control, 2005 and 2005 European Control Conference.CDC-ECC '05.44th IEEE Conference on, pages 7139-7143, 2005.

[12] J. D. Schaffer. Multiple Objective Optimization with Vector Evaluated Genetic Algorithms. In Proceedings of the 1st International Conference on Genetic Algorithms, pages 93-100, NJ, USA, 1985. Lawrence Erlbaum Associates, Inc.

[13] N. Srinvas and K. Deb. Multiobjective Optimization Using Nondominated Sorting in Genetic Agorithm. Evolutionary Computation, 2(3):221-248, 1995.

[14] H. Zamzuri, A. C. Zolotas, and R. M. Goodall. Intelligent Control Approaches for Tilting Railway Vehicles. In 19th IAVSD Symposium 2005, Milan, Itali, September 2005.

[15] H Zamzuri, A C Zolotas, and R. M. Goodall. Optimised intelligent tilt controller scheme using genetic algorithms. In Proc. of International Control Conference (ICC2006), Glasgow,UK, September 2006.

[16] A. C. Zolotas. Advanced control strategies for tilting trains. PhD thesis, Loughborough University, 2002.

[17] A.C. Zolotas and R.M. Goodall. Advanced Control Strategies for tilting railway vehicles. In UKACC, Control 2000, Cambridge, Sept. 2000. 
Table 1. PID-Fuzzy Correction Rule Base

\begin{tabular}{|c|c|c|c|c|}
\hline \multirow[t]{4}{*}{8} & \multicolumn{4}{|c|}{ H.Zamzuri et al } \\
\hline & Table 1. PII & Fuzzy Correcti & n Rule Base & \\
\hline & $\dot{\theta}_{v}^{g y r o} / u^{\prime}$ & Neg & Zero & Pos \\
\hline & $\begin{array}{l}\text { Neg } \\
\text { zero } \\
\text { Pos }\end{array}$ & $\begin{array}{l}\text { TiltClkwm } \\
\text { TiltAclkwM } \\
\text { TiltAclkwM }\end{array}$ & $\begin{array}{l}\text { TiltClkwm } \\
\text { NoChange } \\
\text { TiltAclkwm }\end{array}$ & $\begin{array}{l}\text { TiltClkwM } \\
\text { TiltClkwM } \\
\text { TiltAclkwm }\end{array}$ \\
\hline
\end{tabular}



Table 3. Performance assessment results

\begin{tabular}{llcccc}
\hline Deterministic & $\begin{array}{c}\text { PI } \\
\text { Conven. }\end{array}$ & $\begin{array}{c}\text { PID-Fuzzy } \\
\text { Manual }\end{array}$ & $\begin{array}{c}\text { PID-Fuzzy } \\
\text { (NSGA II) }\end{array}$ & $\begin{array}{c}\text { PID-Fuzzy } \\
\text { (MOGA) }\end{array}$ \\
\hline \multirow{2}{*}{ Lat. accel. } & steady state (\%g) & $\mathrm{n} / \mathrm{a}$ & 9.5 & 9.5 & 9.5 \\
& R.M.S deviation error (\%g) & 5.5 & 3.3 & 3.2 & 3.3 \\
\multirow{5}{*}{ Roll gyro } & 19.5 & 14.8 & 13.3 & 13.6 \\
\multirow{2}{*}{ ct } & peak value (\%g) & 0.03 & 0.03 & 0.03 & 0.03 \\
& R.M.S jerk level (rad/s) & 0.08 & 0.12 & 0.11 & 0.11 \\
& peak value (rad/s) & 10.3 & 9.9 & 8.6 & 9.0 \\
& peak value level (\%g/s) & 71.80 & 64.0 & 56.7 & 58.3 \\
& standing (\% of passengers) & 22.6 & 19.0 & 16.5 & 17.12 \\
& seated (\% of passengers) & & & & \\
& Ride qual. & & & & \\
& Stochastic & 0.381 & 0.381 & 0.371 & 0.377 \\
& passive tilt (\%g) & 0.389 & 0.40 & 0.38 & 0.38 \\
& active tilt(\%g) & 2.15 & 5.6 & 3.12 & 0.67 \\
\hline
\end{tabular}

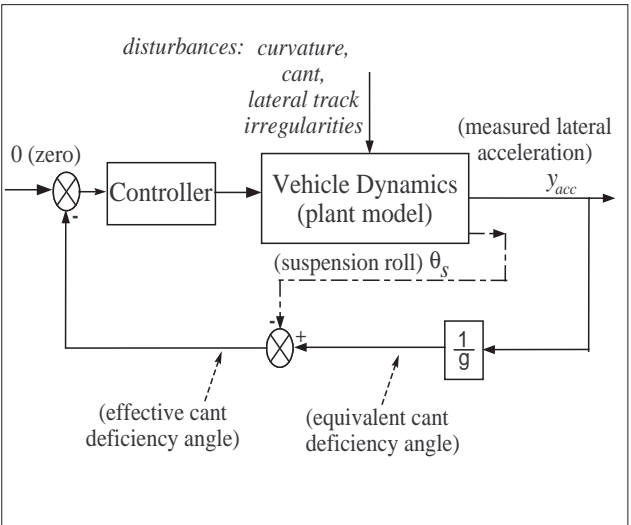

(a) Intuitive partial nulling control (early-type)

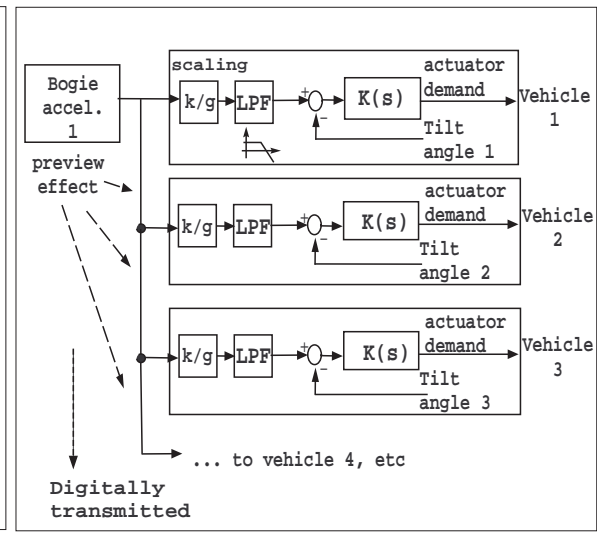

(b) Command driven with precedence control (commercial)

Figure 1. Tilt control approaches 


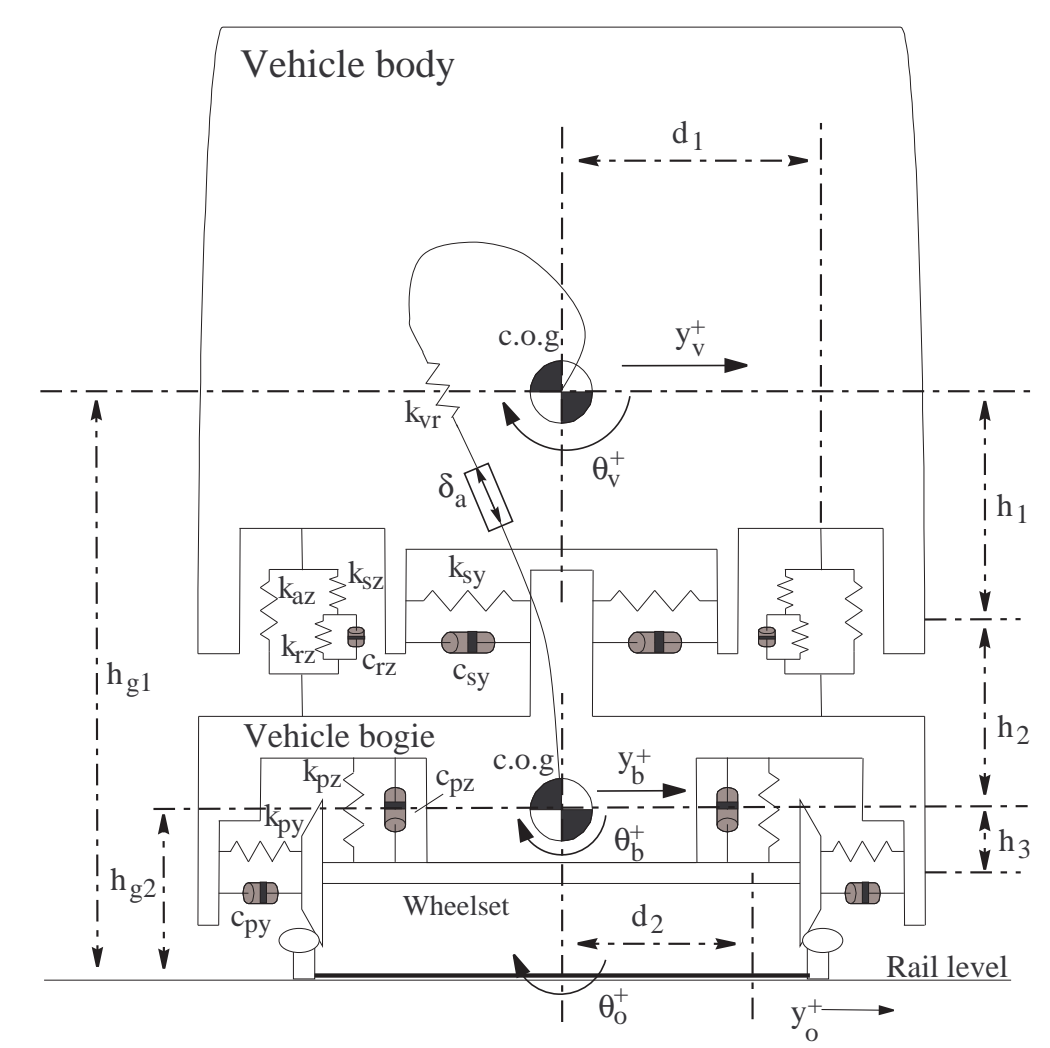

Figure 2. End-view schematic of tilting vehicle

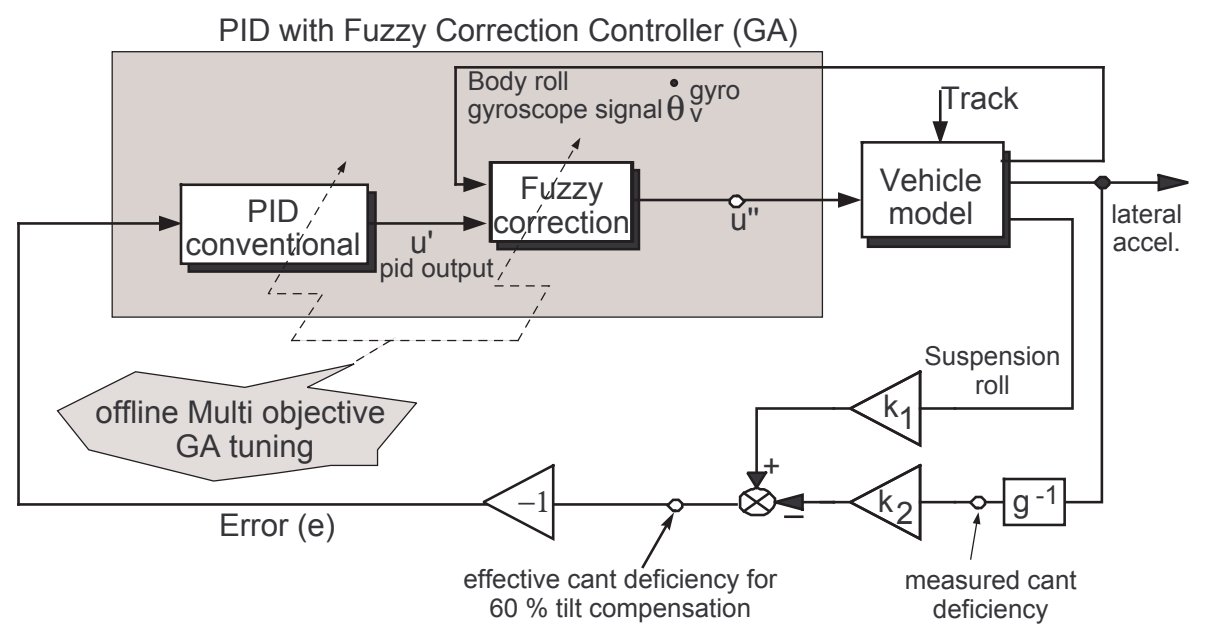

Figure 3. PID with Fuzzy Correction using Multi objective GA tuning method (offline)

$60 \%$ tilt compensation

easured cant 


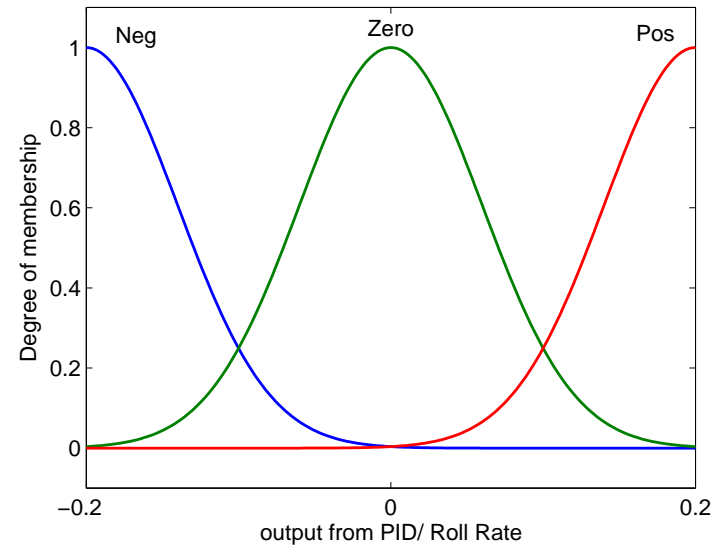

(a) output from PID ( $\left.\mathrm{u}^{\prime}\right)$ and Roll gyro $\left(\dot{\theta}_{v}^{\text {gyro }}\right)$

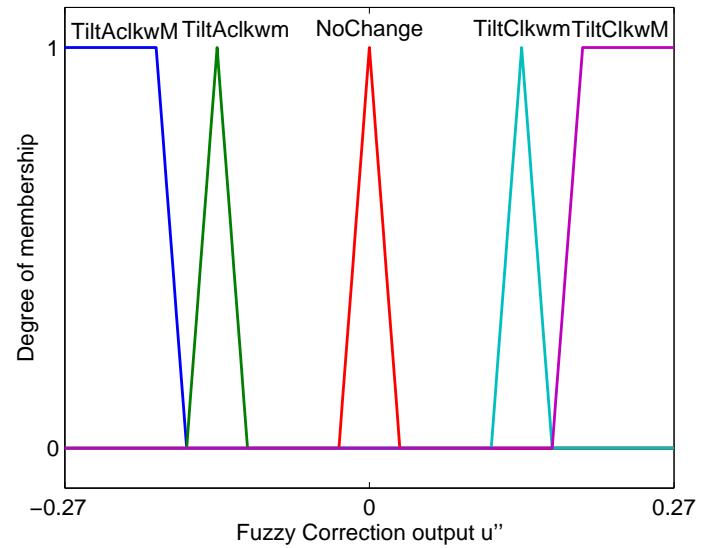

(b) Output controller u"

Figure 4. Input output membership function.

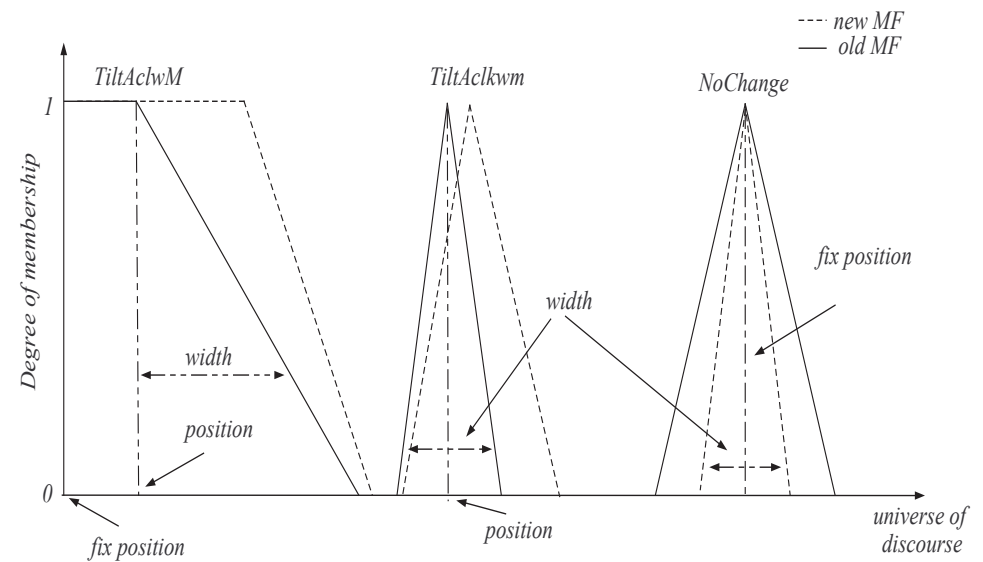

Figure 5. Tuning of position and width of output membership functions

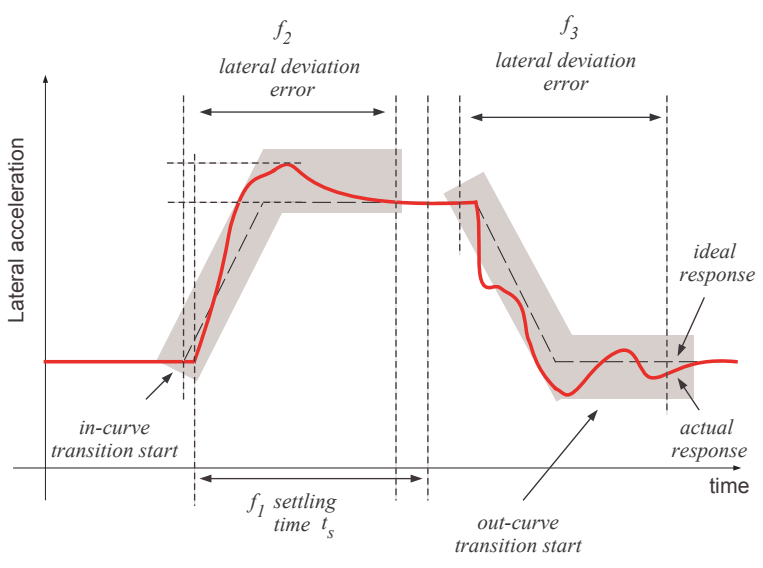

(a)

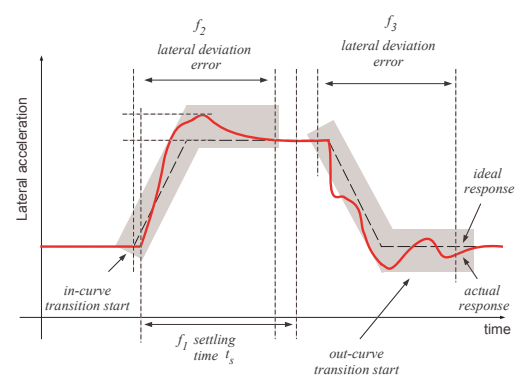

(b)

Figure 6. Training trajectory for (a) lateral acceleration and (b) roll rate angle. 


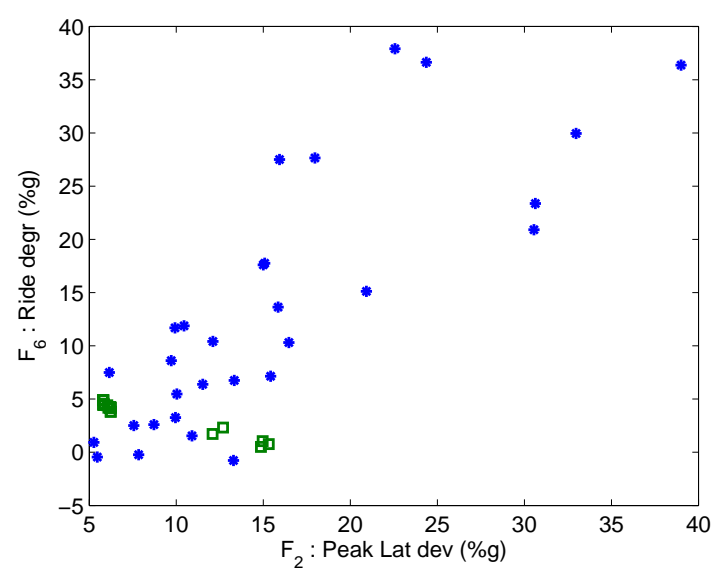

(a) Generation:5

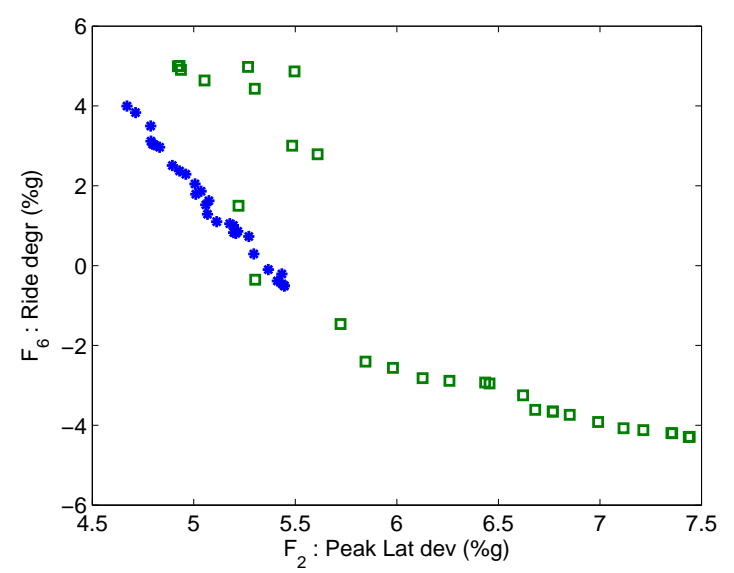

(c) Generation:150

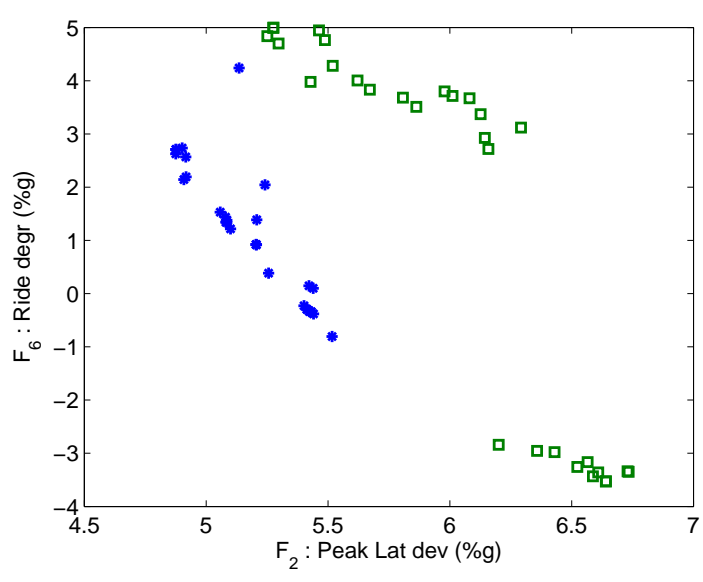

(b) Generation:50

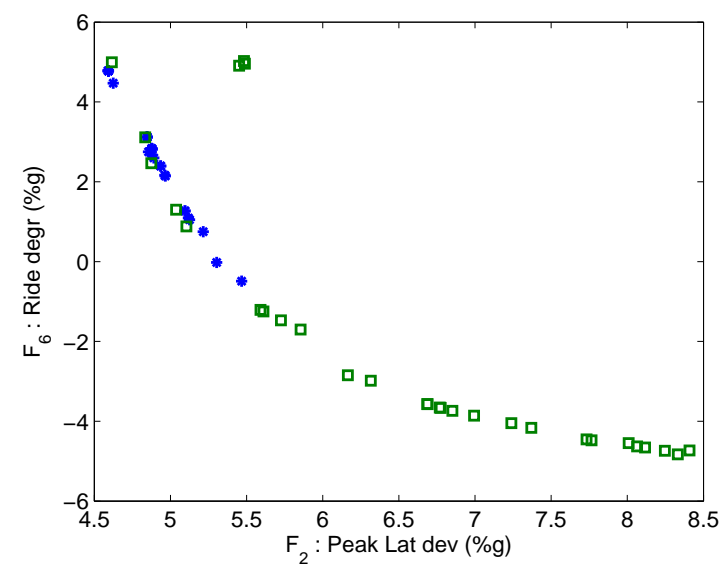

(d) Generation:500

Figure 7. Optimization convergence: MOGA '*', NSGAII ' $\square$ '

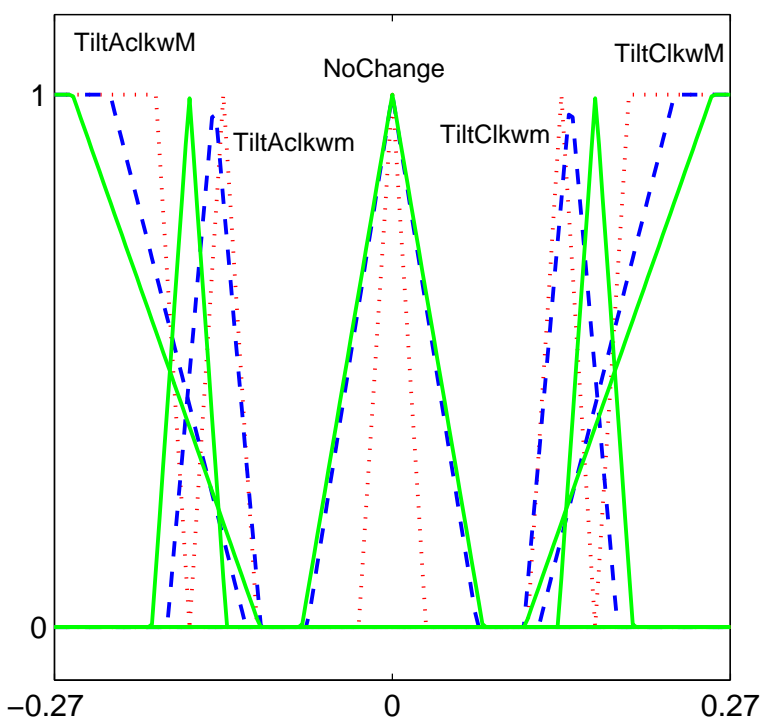

Figure 8. Membership functions manually-tuned (dotted), MOGA (dahsed) and NSGAII (solid). 


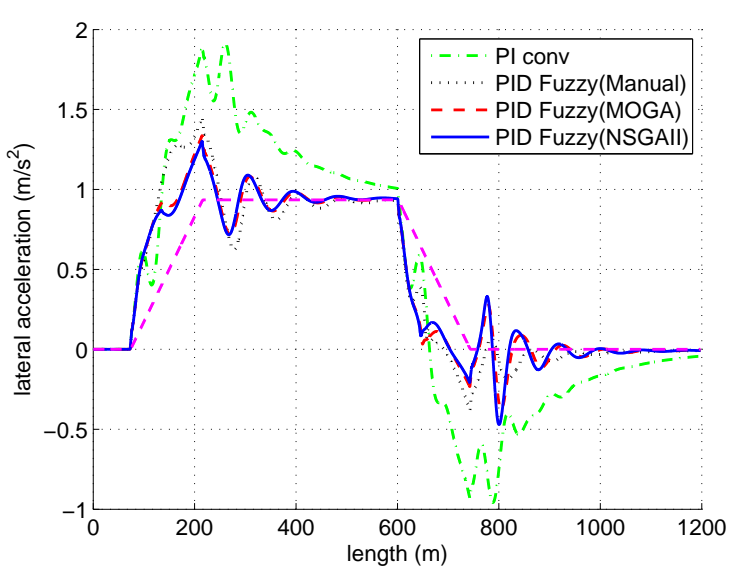

(a) Passenger lateral acceleration

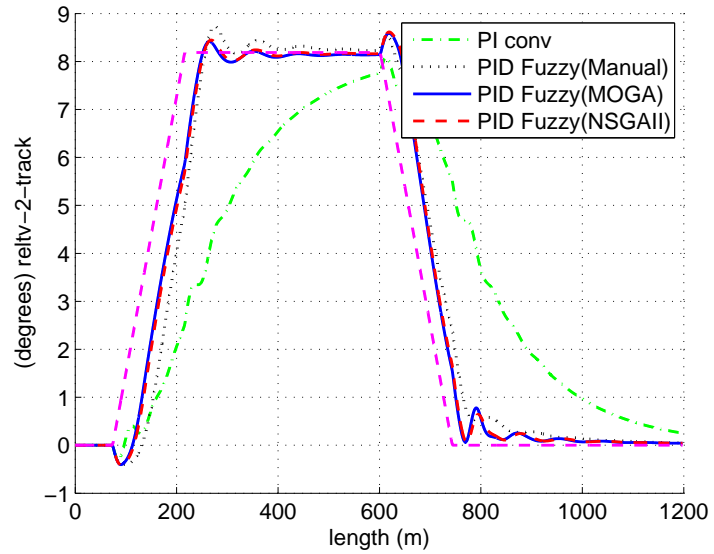

(b) Body roll angle

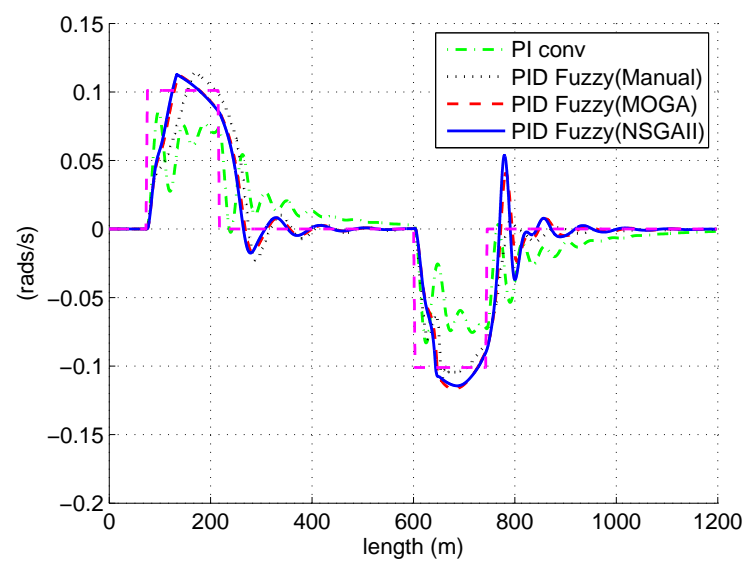

(c) Body Roll gyroscope signal

Figure 9. Output responses 